\title{
Assessment of patient dose in digital systems
}

\author{
Luís Lança and Augusto Silva
}

\begin{abstract}
The assessment of patient dose has gained increased attention, still being an issue of concern that arises from the use of digital systems. The development of digital technology offers the possibility for a reduction of radiation dose around $50 \%$ without loss in image quality when compared to a conventional screen-film system. Digital systems give an equivalent or superior diagnostic performance and also several other advantages, but the risk of overexposure with no adverse effect on image quality could be present.

This chapter refers to the management of patient dose and provides an explanation of dose-related concepts. In this chapter, exposure influence in dose and image representation and the effects of radiation exposure are also discussed.
\end{abstract}

Keywords: Patient dose; Radiography; Digital system; Assessment; Technology; Radiation dose; Radiation exposure; Performance; Overexposure; Adverse effect; Image quality. 